Nature Reviews Neurology 10, 4 (2014); 17 December 2013; doi:10.1038/nrneurol.2013.263; doi:10.1038/nrneurol.2013.264; doi:10.1038/nrneurol.2013.266

\section{IN BRIEF}

\section{HEADACHE}

Norwegian study identifies headache as a risk factor for vascular dementia

A prospective, population-based study involving a cohort of 51,383 individuals from Norway has provided evidence that headache is a risk factor for vascular dementia (VaD) and mixed dementia, but not for Alzheimer disease (AD). The study examined the relationship between headache status (headache-free, any headache, migraine, or nonmigrainous headache) and later development of dementia in participants enrolled in the Nord-Trøndelag Health Study. The researchers found that compared with headache-free individuals, those with any headache at baseline demonstrated a twofold or greater risk of developing VaD or mixed dementia. By contrast, no association was observed between any headache and the risk of developing AD.

Original article Hagen, K. et al. Headache as a risk factor for dementia: a prospective population-based study. Cephalalgia doi:10.1177/0333102413513181

\section{NEURODEGENERATIVE DISEASE}

Histone deacetylase 4 promotes cytoplasmic huntingtin aggregation in mouse models of Huntington disease

In the brains of mice with Huntington disease (HD), the transcriptional repressor histone deacetylase 4 (HDAC4) colocalizes with mutant huntingtin to form cytoplasmic inclusions, according to research reported in PLoS Biology. Mielcarek et al. found that an HDAC4 knock-down in these animals delayed the formation of these inclusions without affecting global transcriptional regulation or nuclear huntingtin aggregation. The knock-down also restored neuronal and synaptic function, ameliorated neurological impairments, and increased survival times. The researchers conclude that HDAC4 could represent a new target for small-molecule therapeutics in HD.

Original article Mielcarek, M. et al. HDAC4 reduction: a novel therapeutic strategy to target cytoplasmic huntingtin and ameliorate neurodegeneration. PLoS Biol. 11, e1001717 (2013)

\section{ALZHEIMER DISEASE}

Primate study deals a fresh blow to the peripheral sink hypothesis

The peripheral sink hypothesis - the idea that levels of soluble amyloid- $\beta(A \beta)$ in the brain and the periphery are in equilibrium, such that peripheral depletion of $A \beta$ should lead to removal of $A \beta$ from the brain-has prompted preclinical trials of $A \beta$-degrading agents. The results to date, however, have been disappointing: one study reported in early 2013 showed that brain levels of $A \beta$ remained unchanged in a mouse model of $A D$ after treatment with the $A \beta$-degrading protease neprilysin. A new study has extended these findings to rats and monkeys. After 1 month of treatment with neprilysin in these animals, peripheral $A \beta$ levels were substantially decreased, but brain and cerebrospinal fluid levels were unaffected. The authors suggest that these findings invalidate the peripheral sink hypothesis.

Original article Henderson, S. J. et al. Sustained peripheral depletion of amyloid- $\beta$ with a novel form of neprilysin does not affect central levels of amyloid- $\beta$. Brain doi:10.1093/brain/awt308

Further reading Walker, J. R. et al. Enhanced proteolytic clearance of plasma $A \beta$ by peripherally administered neprilysin does not result in reduced levels of brain $A \beta$ in mice. J. Neurosci. 33, 2457-2464 (2013) 